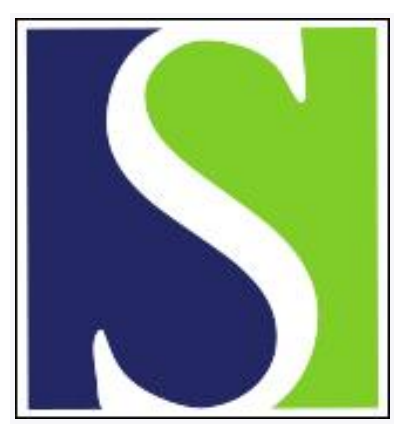

Scand J Work Environ Health 1999;25(2):81-83

https://doi.org/10.5271/sjweh.409

Issue date: Apr 1999

In musculoskeletal epidemiology are we asking the unanswerable in questionnaires on physical load?

by Burdorf $A$, van der Beek AJ

The following articles refer to this text: 2001;27(1):30-40;

2001;27(1):41-48; 2002;28(4):232-237; 2009;35(4):245-260;

2014;40(2):186-194; 2014;40(3):252-265; 2021;47(4):277-286

This article in PubMed: www.ncbi.nlm.nih.gov/pubmed/10360462

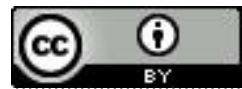




\section{In musculoskeletal epidemiology are we asking the unanswerable in questionnaires on physical load?}

The contribution of physical activity at work to the occurrence of musculoskeletal disorders keeps many researchers busy. It has become almost impossible to keep abreast of the abundant number of publications that explore the question of how physical work load causes and aggravates musculoskeletal disorders. Encouraging progress is being made in understanding which determinants of physical load are associated with the risk of contracting specific musculoskeletal disorders. For back disorders there is ample evidence for a strong association with manual materials handling, frequent bending and twisting, physically heavy work, and whole-body vibration (1). Recent epidemics of carpal tunnel syndrome and repetitive strain injuries in various countries have convincingly demonstrated the negative impact of specific postures and movements on the musculoskeletal system of workers (2).

Despite this knowledge, it is still difficult, however, to quantify the exact levels of physical load at which an increase in musculoskeletal risk occurs. Only in recent years has the quantitative assessment of physical load emerged as a key issue in epidemiologic studies on musculoskeletal disorders. The most widely used method of assessing physical load during work is the self-administered questionnaire, which offers certain benefits over alternative methods such as observations and direct measurements. A variety of reasons can explain the popularity of questionnaires among epidemiologists. Costs of data collection are low, application is feasible in every study design, a large array of different parameters of exposure can be determined simultaneously, and all risk factors are assessed with a similar approach. In the ideal world, direct instrumentation techniques are available to obtain objective information on all relevant aspects of physical load over time for each subject. However, in the reality of research resources, questionnaires sometimes provide the only opportunity to describe the distribution of physical load in the population under study.

The Scandinavian Journal of Work, Environment \& Health has featured prominently in publications addressing the validity of self-reported physical load in epidemiologic research $(3,4,5)$. These publications have taught us that well-designed questionnaires are able to identify relative differences in physical load among occupational groups and, hence, rank these groups as to their level of physical load. In the design of epidemiologic studies, whether a questionnaire has an additional value to available literature or expert judgments must be considered since there is no need for a questionnaire to establish that the tasks of managing directors are physically less demanding than those of nurses. However, most measures of physical load lack sufficient precision and accuracy to present valid estimates of the mean level of physical load for an occupational group. Assessing absolute levels of physical load for individual workers by means of a questionnaire is even more problematic. It is obvious that we simply cannot request a worker to provide precise and accurate answers to the questions "How many times per hour do you have to lift a 5- to 10-kg load on an average workday?" and "How long did you work with your back bent forward more than 45 degrees in the past shift?" Questions with less measurement detail can result in exposure information that is too crude to facilitate application in the study of quantitative exposure-response relationships. Nevertheless, in spite of these apparent drawbacks, several useful applications of questionnaires on physical load have been published. For a group of workers, questionnaires can be helpful to identify important determinants of physical load, which subsequently can be quantified by more objective assessment strategies. General trends over time can be monitored by repeated questionnaires. Workers' self-reports have been used successfully to rank perceived physical 
load in specific activities, tasks, and jobs. The relative ranking of physical load provides important information on the distribution of determinants of physical load in a group of workers with similar exposure profiles. In addition, it can enable evaluation of preventive intervention aimed at large groups of workers.

Given the reluctance to adopt a questionnaire as a valuable tool for objective estimates of physical load, we must ask "Is there a need to develop new questionnaires on physical load in epidemiologic research on musculoskeletal disorders?" The answer is definitely no if these questionnaires are aimed at describing physical load as dichotomous variables or in qualitative terms like low, moderate, and high. Do not waste your time, but rather ask a well-informed colleague for available questionnaires. The answer is certainly yes if the questionnaire, in combination with other assessment tools, will contribute to a quantitative description of the frequency, duration, and amplitude of determinants of physical load.

In this issue of the Scandinavian Journal of Work, Environment \& Health Hollmann and his coworkers publish an interesting approach to the development of a new questionnaire on physical load (6). Self-reports of workers on body postures and the lifting and carrying of loads are used to construct an index for overall physical work load by weighing these self-reports according to their importance in a biomechanical model on compressive forces at the lumbosacral disc. The resulting physical workload index varied little over time, discriminated among workers in four different job categories, and was associated with musculoskeletal complaints. This work essentially introduces a calibration procedure that links subjective assessments in a questionnaire to objective estimates of physical load with more advanced techniques. In a recent study a similar linkage was conducted, and the results showed that, at the group level, the estimates of compression forces, shear forces, and moments at the L4/L5 level of the spine compared well between self-reports and video analysis (7). Such approaches can be extended to other forms of calibration as well. It has been suggested that workers' self-reports on manual materials handling should be evaluated by measurements during task simulation (8). Such external validation with objective measurements can be used to calibrate workers' ratings in a pilot study at the workplace or in laboratory simulation and, subsequently, to collect the subjective ratings by means of a questionnaire in a large epidemiologic study. It must be stressed, however, that these approaches, based on self-reported data, can only yield rather crude estimates of biomechanical load.

In musculoskeletal epidemiology exposure assessment strategy remains a huge challenge. There are no simple solutions since researchers are usually faced with a large array of variables that need to be quantified. The perfect device that captures all relevant dimensions of physical load simultaneously still has to be invented. In general, the assessment calls for creative approaches that combine different methods and measurement techniques. In combination with objective methods, questionnaires can play an essential role in the assessment of physical load at work, as has been demonstrated in recent publications. Epidemiologists and occupational health professionals dealing with work-related musculoskeletal disorders are challenged to convert these new approaches into powerful tools in their daily practice. Although imperfect, information on physical load collected from workers' self-reports has proved to be useful for several epidemiologic studies on musculoskeletal disorders. However, the aim and application of a questionnaire on physical load in epidemiologic research have to be discussed thoroughly. Our experiences can be summarized in the following two basic recommendations: (1) do not ask questions that cannot be answered and (2) do not ask questions for which the answers are already known.

\section{References}

1. Burdorf A, Sorock G. Positive and negative evidence of risk factors for back disorders [review]. Scand J Work Environ Health 1997;23:243--56.

2. Bernard BP. Musculoskeletal disorders and workplace factors: a critical review of epidemiologic evidence for work- 
related musculoskeletal disorders of the neck, upper extremities, and low back. Cincinnati (OH): National Institute for Occupational Safety and Health, 1997.

3. Wiktorin C, Karlqvist L, Winkel J, Stockholm MUSIC I study group. Validity of self-reported exposures to work postures and manual materials handling. Scand J Work Environ Health 1993;19:208-14.

4. Viikari-Juntura E, Rauas S, Martikainen R, Kuosma E, Riihimäki H, Takala E-P, Saarenmaa K. Validity of self-reported physical work load in epidemiologic studies on musculoskeletal disorders. Scand J Work Environ Health 1996;22:2519.

5. Pope DP, Silman AJ, Cherry NM, Pritchard C, Macfarlane GJ. Validity of a self-completed questionnaire measuring the physical demands of work. Scand J Work Environ Health 1998;24:376-85.

6. Hollmann S, Klimmer F, Schmidt K-H, Kylian H. Validation of a questionnaire for assessing physical work load. Scand J Work Environ Health 1999;25:105-14.

7. Andrews DM, Norman RW, Wells RP, Neumann P. The accuracy of self-report and trained observer methods for obtaining estimates of peak load information during industrial work. Int J Ind Ergon 1997;19:445-55.

8. Wiktorin C, Selin K, Ekenvall L, Kilbom $\AA$, Alfredsson L. Evaluation of perceived and self-reported manual forces exerted in occupational materials handling. Appl Ergon 1996;27:231—9.

Alex Burdorf, PhD, Allard J van der Beek, $P h D$

Department of Public Health

Erasmus University Rotterdam

PO BOX 1738

3000 DR Rotterdam, The Netherlands

[E-mail: burdorf@mgz.fgg.eur.nl] 